\title{
Studies of Nucleon Spin Structure at HERMES
}

\author{
Bryan Tipton \\ Kellogg Radiation Laboratory 106-38 \\ 1200 E. California Blvd. \\ Pasadena, CA 91125 \\ E-mail: tipton@krl.caltech.edu \\ On behalf of the HERMES collaboration
}

\begin{abstract}
The HERMES experiment studies the spin structure of the nucleon using polarized semi-inclusive deep-inelastic positron scattering and polarized quasi-real photoproduction. Previous analyses of nucleon spin structure combine inclusive deepinelastic scattering data and measured hyperon decay constants assuming SU(3) flavor symmetry; asymmetries in the processes studied at HERMES may constrain nucleon spin structure more directly. Results are discussed for quark polarizations extracted from semi-inclusive deep-inelastic scattering asymmetries, for gluon polarization extracted from the asymmetry of high- $p_{T}$ hadron pairs, and for the first observation of single-spin azimuthal asymmetries in pion production.
\end{abstract}

\section{INTRODUCTION}

The HERMES experiment studies the spin structure of the nucleon using polarized scattering of electrons and positrons on nucleons:

$$
\vec{l}+\vec{N} \rightarrow l^{\prime}+X .
$$

Previous deep-inelastic scattering (DIS) experiments studied this reaction inclusively, detecting only the final lepton. In these experiments, one is sensitive to the polarized structure functions, $g_{1}(x)$ and $g_{2}(x)$. The function $g_{1}(x)$ possesses a simple interpretation in the Quark-Parton Model(QPM) as the sum over all flavors of polarized parton distribution functions(PDFs), $\Delta q$ :

$$
g_{1}^{p}(x)=\frac{1}{2} \sum_{q} e_{q}^{2}\left(q^{+}(x)-q^{-}(x)\right),=\frac{1}{2} \sum_{q} e_{q}^{2} \Delta q(x) .
$$

The function, $q^{+(-)}$, represents the distribution of quarks of flavor $q$ with spin parallel (anti-parallel) to the nucleon spin, and $e_{q}$ is the quark charge in units

CP539, Symmetries in Subatomic Physics: $3^{\text {nt }}$ Int'l. Symp., edited by X.-H. Guo, A. W. Thomas, and A. G. Williams (C) 2000 American Institute of Physics 1-56396-964-5/00/\$17.00

217 
of elementary charge $|e|$. Here, $x$ is the Bjorken scaling variable $x=Q^{2} / 2 M_{p} \nu$, measured by scattered lepton kinematics; $Q^{2}$ and $\nu$ are the negative squared fourmomentum and energy of the exchanged virtual photon.

One may view the spin of the nucleon in terms of the contributions of its parton constituent:

$$
<s_{z}^{N}>=\frac{1}{2}=\frac{1}{2} \Delta \Sigma+L_{z}^{q}+\Delta G+L_{z}^{G},
$$

where $\Delta \Sigma, \Delta G, L_{z}^{q}$ and $L_{z}^{G}$ are contributions from quark and gluon intrinsic spin and quark and gluon orbital angular momentum. The quark spin contribution, $\Delta \Sigma$, can be further divided among the quark flavors, and between valence and sea contributions:

$$
\Delta \Sigma=\Delta u_{v}+\Delta d_{v}+\Delta u_{s}+\Delta \bar{u}+\Delta d_{s}+\Delta \bar{d}+\Delta s+\Delta \bar{s} .
$$

By measuring $g_{1}(x)$ from various targets and integrating over $x$, it has been found that $\Delta \Sigma$ accounts for only about a third of the nucleon spin, and that the strange sea seems negatively polarized. However, the inclusive DIS data on proton and neutron targets do not constrain $\Delta \Sigma$ alone. In reaching this conclusion, one typically combines polarized DIS results with information from hyperon beta decay constants. SU(3) flavor symmetry in the strong interaction is a key assumption: one assumes hyperon data may be related to nucleon structure through a simple rotation in flavor space.

The HERMES experiment attempts to make a detailed study of nucleon spin structure. The approach taken by HERMES is to measure the spin dependence of all high energy lepton scattering final states with a large acceptance detector. A combined analysis of multiple cross-sections, each having different sensitivity to nucleon spin structure, allows a more complete study of the proton spin in one experiment alone. Symmetries of the strong interaction are then no longer assumed, but rather are tested in the course of the investigation. Three results are discussed here. By measuring semi-inclusive hadron production yields and fitting their double-spin asymmetry in the QPM, the flavor dependence of the quark polarizations may be extracted. Photoproduction of high- $p_{T}$ hadron pairs yields a negative asymmetry, which simulations show is strongly correlated to the polarization of gluons. Finally, single-spin azimuthal asymmetries in pion production hint at a large transversity distribution in the nucleon.

\section{THE HERMES EXPERIMENT}

The HERMES experiment was designed to optimize semi-inclusive DIS studies [1]. The experiment is located at the DESY laboratory in Hamburg, Germany, where a $27.5 \mathrm{GeV}$ positron (or electron) beam circulates in the HERA beam. The positron beam is naturally polarized transverse to the beam direction due to an asymmetry in the emission of synchrotron radiation, and longitudinal polarization 
at the HERMES interaction point is provided by a pair of matched spin rotators. Beam polarizations averaged $55 \%$ during the run, with a relative systematic uncertainty in the measurement of $4.0 \%(3.4 \%)$ for the ${ }^{3} \mathrm{He}(\mathrm{H})$ data.

The HERMES experiment consists of an internal gas target assembly in place along the East Hall interaction point (IP) of the HERA positron storage ring. The HERA beam passes through the center of a $40 \mathrm{~cm}$ long storage cell containing polarized ${ }^{3} \mathrm{He},{ }^{1} \mathrm{H}$, and ${ }^{2} \mathrm{H}$. Two different target technologies inject the nuclei into the cell. In 1995, a ${ }^{3} \mathrm{He}$ target was used, where atoms became polarized through metastability-exchange optical pumping, giving an average polarization of 46\%. [5] An Atomic Beam Source(ABS) used permanent sextupole magnets and radio-frequency units to select desired hyperfine states of ${ }^{1} \mathrm{H}$ in 1996 and 1997 . This source continues to operate with polarized ${ }^{2} \mathrm{H}$ today. Proton polarization averaging $86 \%$ was monitored by a Breit-Rabi polarimeter. [6]

Extending 8.5 meters behind the target, a forward acceptance spectrometer captures the high energy particles produced in the positron-gas interaction. Tracking chambers placed before and after a dipole magnet determine momenta and trajectories of those particles. A threshold Cerenkov detector, a Transition-Radiation Detector, a Preshower Counter, and an Electromagnetic Calorimeter allow separation of electrons from hadrons. The Čerenkov further provides clean determination of pions above particle momenta of 5.6 and $3.8 \mathrm{GeV} / \mathrm{c}$ in 1995 and 1996-7. Before the 1998 running, this Cerenkov was replaced with a Ring-imaging Čerenkov (RICH), which will provide $\pi, K$, and $p$ separation over the entire HERMES kinematic range.

\section{THE POLARIZATION OF QUARKS}

In polarized deep-inelastic scattering, one can construct a cross-section asymmetry of parallel and anti-parallel orientations of the nucleon spin to the lepton beam spin, $A_{\|}$:

$$
A_{\|}=\frac{\sigma^{\uparrow \Downarrow}-\sigma^{\uparrow \Uparrow}}{\sigma^{\uparrow \Downarrow}+\sigma^{\uparrow \Uparrow}}
$$

Under the assumption that $g_{2}(x)$ is zero, the measured double-spin asymmetry directly relates to the asymmetry in nucleon virtual photoabsorption, $A_{1}(x)$, and to the structure function ratio; $\frac{g_{1}}{F_{1}}(x)$ :

$$
A_{1}^{h}=\frac{g_{1}^{h}}{F_{1}^{h}}=\frac{A_{\|}^{h}}{D(1+\gamma \eta)} .
$$

where $h$ includes both inclusive positron data and coincident positive and negative unidentified hadrons, and $D, \gamma$, and $\eta$ are kinematic factors.

In the QPM and under the assumption of factorization, one sees that the photoabsorption asymmetry probes the helicity difference parton distributions $\Delta q(x)$ : 


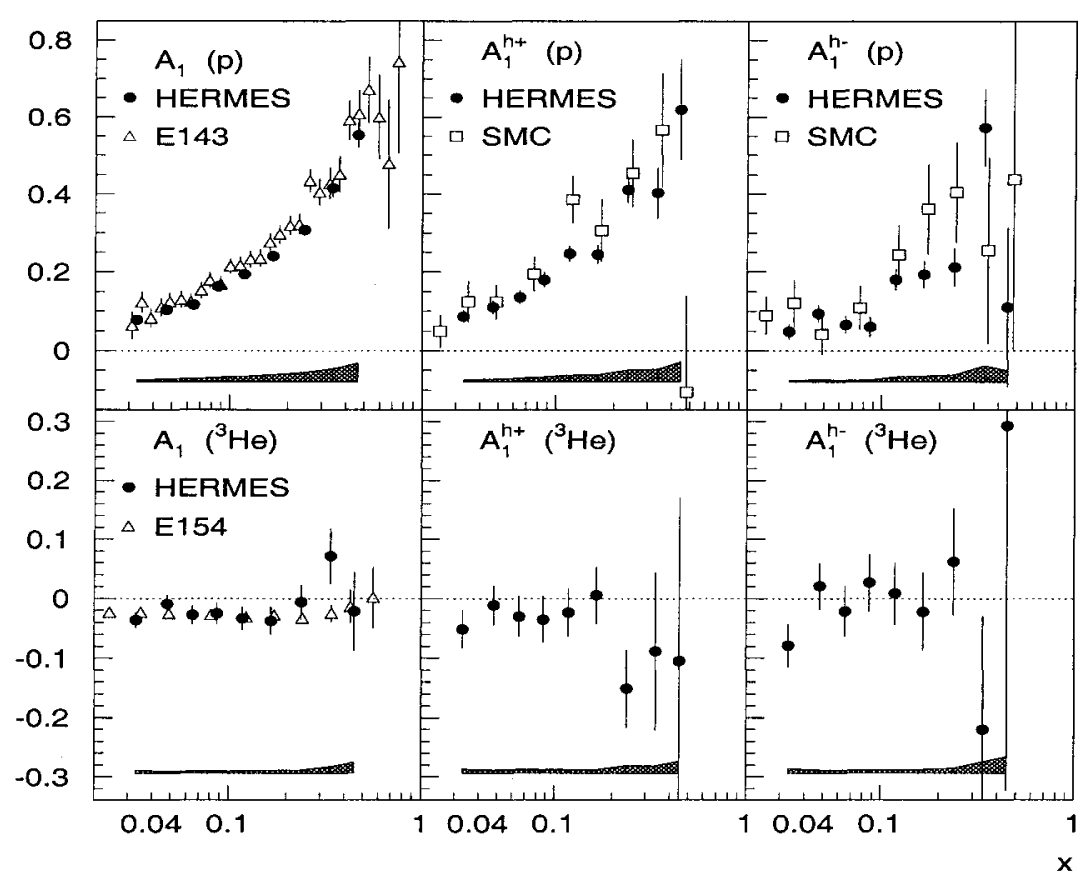

FIGURE 1. Results for the virtual photon asymmetries from HERMES 1995-7 data. The columns show the inclusive, semi-inclusive $h^{+}$, and semi-inclusive $h^{-}$asymmetries while the rows correspond to the ${ }^{1} \mathrm{H}$ and ${ }^{3} \mathrm{He}$ target. The inclusive asymmetries are compared with the total uncertainties of the SLAC E143 [7] and E154 [8] $g_{1} / F_{1}$ measurements. For the proton semi-inclusive asymmetries, a comparison with the SMC [9] results are shown. All points are plotted at measured $x$ and $Q^{2}$.

$$
A_{1}^{h}(x, z)=\frac{\sum_{q} e_{q}^{2} \Delta q(x) D_{q}^{h}(z)}{\sum_{q} e_{q}^{2} q(x) D_{q}^{h}(z)} \frac{\left(1+R\left(x, Q^{2}\right)\right)}{1+\gamma^{2}}
$$

The $D_{q}^{h}(z)$ are the (spin-independent) fragmentation functions. In this model, each measured asymmetry has a different correlation to the underlying quark polarization through the charge and fragmentation function factors. By fitting several of these asymmetries on proton and effective neutron targets, one may extract the polarized quark distributions $\Delta q(x)$.

HERMES has measured asymmetries for inclusive and semi-inclusive $\mathrm{h}^{+}$and $\mathrm{h}^{-}$ reactions from ${ }^{3} \mathrm{He}$ and ${ }^{1} \mathrm{H}$ targets [2]. These asymmetries are shown in Figure 1. The inclusive DIS sample was determined by cuts on $Q^{2}>1 \mathrm{GeV}^{2}, y=\frac{\nu}{E}<0.85$, and the final photon-nucleon center-of-mass energy, $W^{2}>4 \mathrm{GeV}^{2}$. The sizes of the inclusive event samples satisfying these requirements were $2.2 \cdot 10^{6}$ and 2.3 . $10^{6}$ events for ${ }^{3} \mathrm{He}$ and ${ }^{1} \mathrm{H}$ respectively. The analyzed semi-inclusive events form a subsample having a detected charged hadron and $W^{2}>10 \mathrm{GeV}^{2}$. Cuts on the hadron energy fraction, $z>0.2$, and the Feynman variable, $x_{f}>0.1$, also select 
nucleon fragments from the current region. Beam and target polarization measurement uncertainties, uncertainties in $R\left(x, Q^{2}\right)$, and experimental yield fluctuation uncertainties in 1995 are the dominant contributions to the systematic uncertainty of the measured asymmetries.

One may simply rearrange of Equation 7 to separate the quark polarizations from unpolarized physics factors summarized by the purity, $P_{q}^{h}(x)$ :

$$
A_{1}^{h}(x)=\sum_{q} P_{q}^{h}(x) \cdot\left(\frac{\Delta q(x)}{q(x)}\right) \frac{1+R}{1+\gamma^{2}} .
$$

The resulting system of equations may be written in matrix form as,

$$
\overrightarrow{\mathbf{A}}(x)=\mathbf{P} \cdot \overrightarrow{\mathbf{Q}}(x) .
$$

The quark polarizations are obtained by least squares minimization of this equation.

The quark purities relevant for the HERMES experiment have been estimated with a Monte Carlo simulation of unpolarized DIS, based on LUND string fragmentation, [11] CTEQ low $\mathrm{Q}^{2}$ unpolarized parton distributions, [12] and a model of the HERMES detector. ${ }^{3} \mathrm{He}$ asymmetries convert to effective neutron asymmetries through nuclear corrections. [13]

In solving Equation 9, one would like to separate the spin contributions of three quark flavors $u, d$, and $s$, and their anti-quarks $\bar{u}, \bar{d}$, and $\bar{s}$. In practice, limited statistics and sensitivity to the sea quark flavors require selection of a model of average sea polarization. A simple assumption of flavor independent sea polarization is used here:

$$
\frac{\Delta q_{s}}{q_{s}} \equiv \frac{\Delta d_{s}}{d_{s}}=\frac{\Delta u_{s}}{u_{s}}=\frac{\Delta \bar{d}}{\bar{d}}=\frac{\Delta \bar{u}}{\bar{u}}=\frac{\Delta \bar{s}}{\bar{s}}=\frac{\Delta s}{s} .
$$

Note that with further data-taking, this relation may be relaxed in the future and allowing a study of the symmetries implied.

Using the six experimental asymmetries shown in Figure 1, three quark polarizations have been extracted: $(\Delta u+\Delta \bar{u}) /(u+\bar{u})(x)$, the total up flavor polarization; $(\Delta d+\Delta \bar{d}) /(d+\bar{d})(x)$, the total down flavor polarization; and $\left(\Delta q_{s} / q_{s}\right)(x)$, the average polarization of the quark sea. After multiplying by $x$ and the unpolarized PDF parameterization, Figure 2 shows the results from this extraction in nine $x_{B j}$ bins. For $x>0.3$, it is assumed that sea polarization does not contribute significantly to the measured asymmetries; the sea polarization, $\frac{\Delta q_{s}}{q_{s}}(x)$, is set to zero with a small systematic uncertainty. Systematic uncertainties in the extraction originate in the asymmetries' uncertainty and in a study of the sensitivity of the fit to variations in the unpolarized Monte Carlo model.

In Figure 2, the parameterizations of De Florian et. al. $(0.1<\Delta G<0.8, \mathrm{LO})$ [15] Gehrmann and Stirling (Gluon A, LO), [17] and Glück et. al. (Standard, LO) $[16]$ are provided for comparison. A correction of $1+R$ to the De Florian and 
TABLE 1. The first moments of the extracted polarized parton distributions. The results are given at $\mathrm{Q}^{2}=2.5 \mathrm{GeV}^{2}$ for the measured region $0.023<x<0.6$, for the low- $x$ extrapolation and for the total integral. Note that the values for $\Delta s+\Delta \bar{s}$ relies on the assumption in Eq. (10).

\begin{tabular}{|c||c|r|r|r|c|}
\hline & measured region & low- $x$ & \multicolumn{1}{c}{ total integral } & \multicolumn{1}{|c|}{ (3) prediction $[18]$} & $\mathrm{Q}^{2}$ \\
\hline$\Delta u+\Delta \vec{u}$ & $0.51 \pm 0.02 \pm 0.03$ & 0.04 & $0.57 \pm 0.02 \pm 0.03$ & $0.66 \pm 0.03$ & 2.5 \\
$\Delta d+\Delta \bar{d}$ & $-0.22 \pm 0.06 \pm 0.05$ & -0.03 & $-0.25 \pm 0.06 \pm 0.05$ & $-0.35 \pm 0.03$ & 2.5 \\
$\Delta s+\Delta \bar{s}$ & $-0.01 \pm 0.03 \pm 0.04$ & 0.00 & $-0.01 \pm 0.03 \pm 0.04$ & $-0.08 \pm 0.02$ & 2.5 \\
\hline
\end{tabular}

Glück parameterizations is necessary to achieve consistency in model assumptions. The HERMES data agrees with these parameterizations within uncertainties.

Moments of the quark distributions in the measured $x$ range are determined from the area under the measured points. For comparison to previous measurements, the distributions are extrapolated to low $x$ by constant fits to the data; the low $x$ extrapolation is quoted but no value is quoted for the uncertainty due to theoretical ambiguities. The resulting measured region integrals are listed in Table 1. The integrals are compared to predictions from Reference [18], which are corrected to fourth order in QCD; these predictions have been extracted from inclusive data

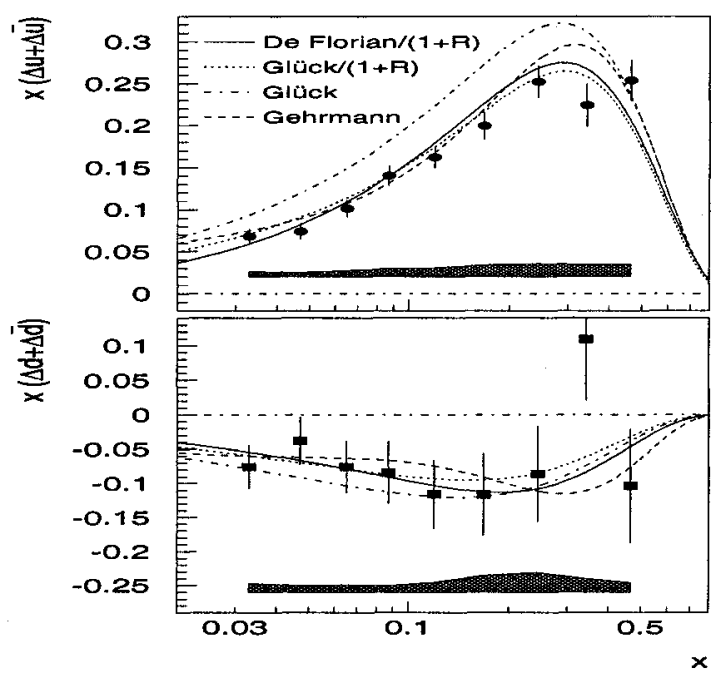

FIGURE 2. Polarized parton distributions for up and down quarks, $x(\Delta u+\Delta \bar{u})$ and $x(\Delta d+\Delta \bar{d})$, compared to parameterizations of world data. The data points have been evolved to $Q^{2}=2.5 \mathrm{GeV}^{2}$ for the comparison. The parameterizations are explained in the text. 
assuming $\mathrm{SU}(3)_{\text {flavor }}$ symmetry. The HERMES values are slightly smaller in magnitude than these predictions, and in particular the sea is unpolarized in the fit. Within uncertainties though, the results are not yet in disagreement.

\section{THE ROLE OF THE GLUON SPIN}

As the contributions of the quarks' intrinsic spins does not sum to one half, the remaining angular momentum inside the proton must lie elsewhere. Significant theoretical activity has focused on a possible large gluon contribution to the proton spin. Attempts have been made to constrain this contribution by fitting the $Q^{2}$ dependence of the inclusive polarized structure functions with a DGLAP formalism. These efforts yield an indication of positively polarized glue [9], but the statistics of the polarized measurements are as yet too poor to produce a definitive constraint.

New generations of polarized experiments study the gluon contribution by examining the polarization dependence of processes directly sensitive to the gluon distribution in the nucleon. One process is the photoproduction of two hadrons at large transverse momentum, $p_{T}$. If the cut on the transverse momentum is sufficiently large, one may reject tree-level quark scattering events with small $p_{T}$ and isolate events from the hard photon-gluon fusion process [10].

HERMES has measured the double-spin asymmetry in the virtual photoproduction of high- $p_{T}$ hadrons on a proton target [3]. Events were selected to have one positively and one negatively charged hadron with total momenta greater than 4.5 $\mathrm{GeV} / \mathrm{c}$ in the HERMES spectrometer. The scattered positron was not required to be detected, and simulations show that very low $Q^{2}$ is probed. Further cuts required the reconstructed invariant mass of the hadrons to be above the $\rho$ resonance. The asymmetry is presented as a function of hadron $p_{T}$ in Figure 3 . In each panel in this figure, a hard cut of $1.5 \mathrm{GeV} / \mathrm{c}$ is placed on the $p_{T}$ of one charged hadron, while the $p_{T}$ of the other is varied. One sees a substantial negative asymmetry when the hard cut is placed on the negative hadron. A much smaller effect is seen in the positive hadron case. If one treats the charged hadron dependence uniformly and averages the plots in Figure 3, one gets the asymmetry in Figure 4.

To relate these asymmetries to gluon polarization, a simulation of the underlying physics processes is required. The PYTHIA Monte Carlo is used to estimate the contribution of various processes to the high- $p_{T}$ events at HERMES. The processes included vector meson production, the QCD Compton effect, and the photon-gluon fusion. These studies indicate that the cross-section in the region of the negative asymmetry is dominated by photon-gluon fusion. As the photon-gluon fusion asymmetry is directly proportional to $\Delta G / G$, this quantity may be extracted from the data, with a small positive background asymmetry from the QCD Compton effect subtracted. It is important to note that while one expects a negative asymmetry from the photon-gluon fusion process, other processes on a proton target yield either a positive or zero asymmetry. The expected asymmetry at HERMES for three $\Delta G / G$ assumptions are also shown in Figure 4. 

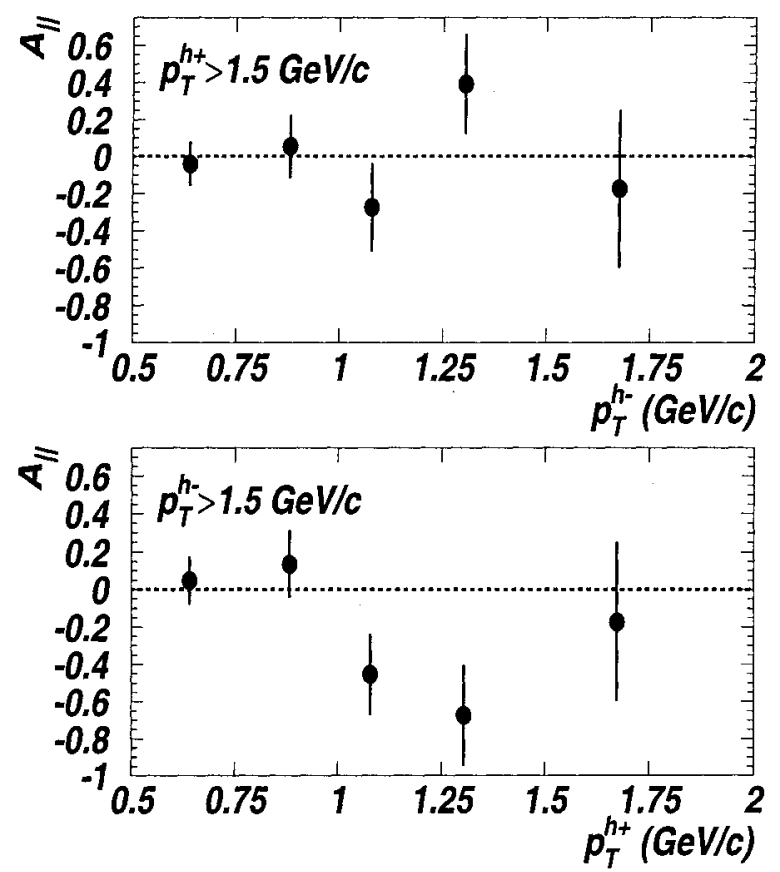

FIGURE 3. The virtual photoproduction asymmetry of high- $p_{T}$ hadron pairs versus the positive hadron $p_{T}$ (top) and the negative hadron $p_{T}$ (bottom).

The points in the region $p_{T}^{h 1}>1.5 \mathrm{GeV} / c$ and $p_{T}^{h 2}>1.0 \mathrm{GeV} / c$ give an average asymmetry of $A_{\|}=-0.28 \pm 0.12 \pm 0.02$. Using the above Monte Carlo analysis, this asymmetry yields value of $\Delta G / G$ in LO QCD as $0.41 \pm 0.18$ (stat.) \pm 0.03 (syst.). Only experimental systematics are quoted.

\section{SINGLE-SPIN AZIMUTHAL ASYMMETRIES}

While the above results probe the structure of the parton spin in along the axis of the proton momentum, the transverse parton spin remains largely unexplored. The structure function, $h_{1}(x)$, and distribution functions, $\delta q(x)$, associated with transversity are as fundamental in QCD as their longitudinal partners, $g_{1}(x)$ and $\Delta q(x)$. Study of these distributions involves an examination of rotational symmetry in the nucleon. In the non-relativistic limit, one expects that no direction in space is preferred in a nucleon, and therefore $\Delta q(x)$ and $\delta q(x)$ are equal. With relativity, the two distributions may diverge from each other.

Unfortunately, $\delta q(x)$ are chiral odd distributions which are then unobservable in deep-inelastic scattering. One can circumvent this limitation by seeking observables in which the chiral odd distributions are associated with chiral odd fragmentation functions. Final state interactions in fragmentation may allow sensitivity to the spin 


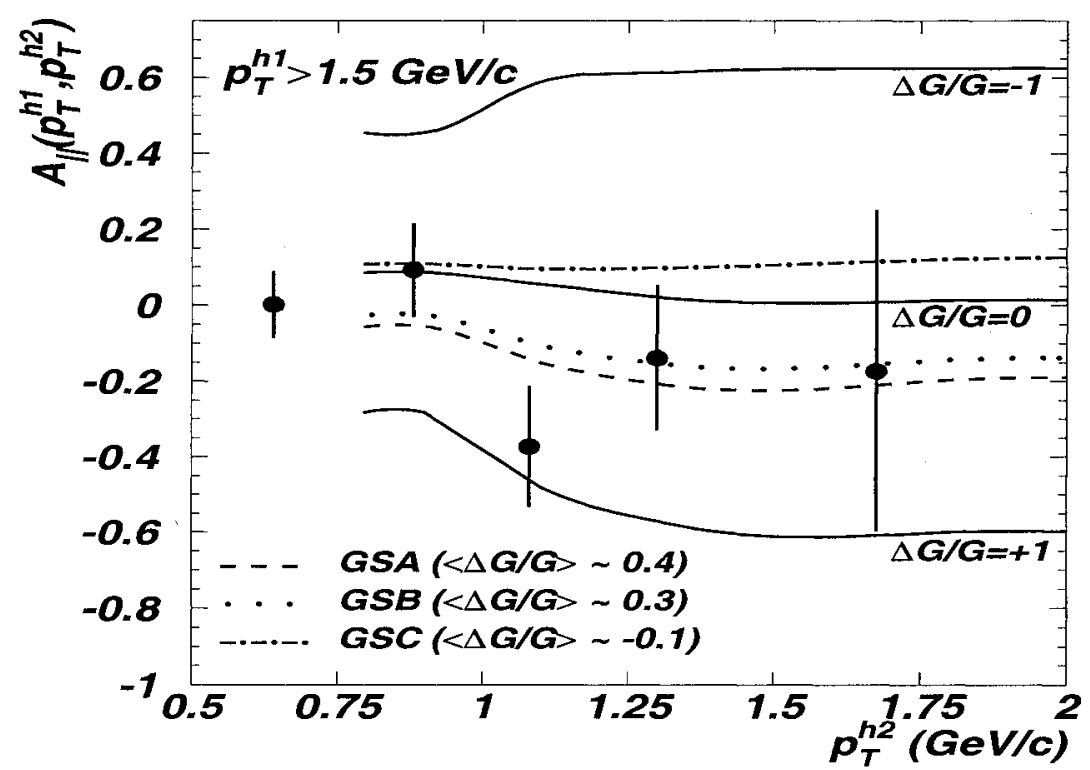

FIGURE 4. The virtual photoproduction asymmetry of high- $p_{T}$ hadrons compared to Monte Carlo predictions for various $\Delta G / G$ assumptions. The dotted lines represent the predictions of reference [17].

transversity. Single-spin azimuthal asymmetries in semi-inclusive pion production are such observables.

HERMES has measured semi-inclusive pion single-spin asymmetries using a polarized proton target [4]. By weighting the cross-section by a sinusoid function, $W(\phi)$, the analyzing powers $\mathrm{A}_{U L}^{W(\phi)}$ may be determined. In Figure 5 , these analyzing powers are shown versus $x$ for $\pi^{+}$and $W(\phi)=\sin \phi$ and $\sin 2 \phi$. For $\pi^{+}$one sees a strong rising $x$ dependence in the $\sin \phi$ moment, similar in shape to the rise in the $u$-quark longitudinal polarization in Figure 2. The corresponding $\pi^{-}$moment (not shown) is consistent zero. This may be an indication of flavor dependence of the moment, as the $\pi^{+}$production is dominated by $u$-quark scattering while the $\pi^{-}$production has more $d$-quark contributions.

Note that though the proton target was longitudinally polarized, transverse spin effects can still manifest in the data as the virtual photon direction and the proton spin are not collinear for small $Q$. One expects to see a larger effect from a transversely polarized target. The $\sin 2 \phi$ analyzing power, as well as the beam spin correlated analyzing powers $\mathrm{A}_{L U}^{W(\phi)}$, have also been investigated, and are found consistent with zero. 


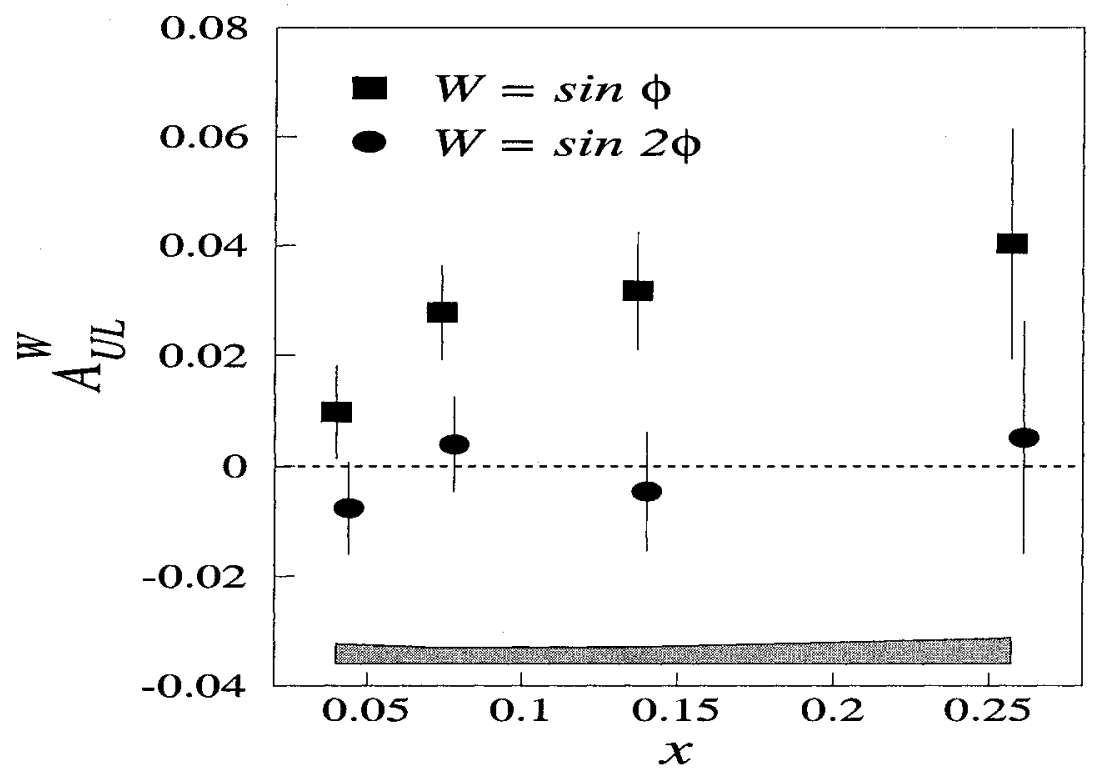

FIGURE 5. Moments of single-spin pion azimuthal asymmetries on a polarized proton target.

\section{CONCLUSIONS AND OUTLOOK}

The first three years of HERMES data taking have yielded high statistics polarized lepton scattering data at $27.5 \mathrm{GeV}$ on ${ }^{3} \mathrm{He}$ and ${ }^{1} \mathrm{H}$ targets. The polarized measurements yield inclusive and semi-inclusive charged hadron asymmetries, which have been presented as a function of $x$. In the framework of the Quark-Parton Model, these asymmetries are simultaneously fitted to yield helicity distributions for up, down and sea quarks. The current data set begins to test $\mathrm{SU}(3)_{\text {flavor }} \mathrm{sym-}$ metry in the strong interaction, but no significant breaking is seen yet. Gluon polarizations are studied by looking at the asymmetry in high- $p_{T}$ hadron pairs; an indication of a positive glue is seen. Azimuthal asymmetries may explore the properties of the nucleon spin under spatial rotation. The first single-spin azimuthal pion production asymmetry in semi-inclusive DIS is seen.

HERMES will soon have a new data set with high statistics on a deuterium target, which will significantly improve the precision on the $d$-quark helicity distribution. Further sensitivity to sea polarizations is gained by identification of different coincident hadron species. Before the deuterium run began, the Threshold Čerenkov was replaced with a Ring Imaging Čerenkov(RICH), which may identify $\pi, K$ and $p$ particles over nearly all momenta accepted by the spectrometer. After 2000, HERMES will use a transversely polarized ${ }^{1} \mathrm{H}$ and ${ }^{2} \mathrm{H}$ target in order to study spin transversity in more detail. 


\section{ACKNOWLEDGMENTS}

The author would like to acknowledge the support of the U.S. National Science Foundation.

\section{REFERENCES}

1. Ackerstaff, K., et al., Nucl. Instrum. Methods A 417, 230 (1998).

2. Ackerstaff, K., et al., Phys. Lett. B 464, 123 (1999).

3. Airapetian, A., et al., Phys. Rev. Lett. 84, 2584 (2000).

4. Airapetian, A., et al., Phys. Rev. Lett. 84, 4047 (2000).

5. De Schepper, D., et al., Nucl. Instrum. Methods A 419, 16 (1998).

6. Braun, B., in Proc. of the Workshop "Polarized gas targets and polarized beams", ed. R.J. Holt and M.A. Miller, AIP Conf. Proc. 42169 (1997).

7. Abe, K., et al., Phys. Rev. D 58, 112003 (1998).

8. Abe, K., et al., Phys. Rev. Lett. 79, 26 (1997).

9. Adeva, B., et al., Phys. Rev. D 58, 112001 (1998).

10. Bravar, A., von Harrach, D., and Kotzinian, A., Phys. Lett. B 421, 349 (1998).

11. Andersson, B., et al., Phys. Rept. 97, 33 (1983).

12. Lai, H.L., et al., Phys. Rev. D 126, 1280 (1997).

13. Friar, J.L., et al., Phys. Rev. C 42, 2310 (1990).

14. Glück, M., et al., Z. Phys. C 67, 433 (1995).

15. De Florian, D., et al., Phys. Rev. D 57, 5803 (1998).

16. Glück, M., et al., Phys. Rev. D 53, 4775 (1996).

17. Gehrmann, T. and Stirling, W. J., Phys. Rev. D 53, 6100 (1996).

18. Ellis, J. and Karliner, M., Invited Lectures at the International School of Nucleon Spin Structure, Erice, August 1995, hep-ph/9601280. 